\title{
RDUS
}

Revue de DROIT

UNIVERSITÉ DE SHERBROOKE

Titre : $\quad$ Note bibliographique

LES FAITS ALTERNATIFS DU DROIT CONSTITUTIONNEL

Auteur(s) : $\quad$ René PÉPIN

Revue: $\quad$ RDUS, 2017, volume 47, numéro 2-3

Pages: $\quad 473-474$

ISSN : $\quad 0317-9656$

Éditeur : $\quad$ Université de Sherbrooke. Faculté de droit.

URI : $\quad$ http://hdl.handle.net/11143/16167 
Page vide laissée intentionnellement. 


\section{LES FAITS ALTERNATIFS DU DROIT CONSTITUTIONNEL*}

par René PEPIN ${ }^{* *}$

Nous avons ici une des présentations faites à l'occasion des conférences annuelles Chevrette-Marx, consacrées soit aux principes fondamentaux, soit au fédéralisme ou encore aux libertés publiques.

Le titre de l'ouvrage de Danielle Pinard, Les faits alternatifs $d u$ droit constitutionnel, nous amène à penser aux interventions devant les médias de Kellyanne Conway, conseillère de l'actuel président des États-Unis. L'auteure y fait allusion pour expliquer que ce sont là des éléments faisant appel à l'émotion et à des opinions personnelles plutôt qu'à des faits vérifiables. Elle se pose ainsi les questions suivantes: Les faits sur lesquels les lois s'appuient souvent sont-ils véridiques? Les conclusions que les législateurs tirent de "faits " pourraient-elles n'être que de simples approximations?

Le questionnement de l'auteure s'avère pertinent parce qu'en droit constitutionnel on semble être passé d'un extrême à l'autre. Jusqu'aux années 60, les tribunaux acceptaient en preuve presque uniquement le texte de la loi. Même la lecture du Hansard était suspecte, parce qu'il fallait trouver l'intention du Parlement qui avait adopté la loi, et non l'opinion d'une seule personne, même si c'était le ministre qui avait parrainé le projet. Toutefois, les tribunaux n'ont plus le choix de nos jours. La Constitution canadienne dit que les parlements peuvent retreindre un droit fondamental, pourvu que cette limite soit raisonnable dans une société libre et démocratique. Il faut alors vérifier s'il y a consensus, chez les historiens, sociologues, éthiciens ou politologues, sur le caractère raisonnable d'une mesure. Or souvent ces " preuves " ne sont pas accessibles, et ce, pour diverses raisons : les sciences sociales ne se sont pas

*. Danielle PINARD, Les faits alternatifs du droit constitutionnel, Montréal, Les Éditions Thémis, 2018, 57 p.

**. Professeur titulaire, Faculté de droit, Université de Sherbrooke. 
posé ces questions, les résultats sont encore provisoires ou bien les études se contredisent.

Le sujet des faits alternatifs n'est pas nouveau. Il a déjà été traité ailleurs. Cependant, l'auteure l'examine en profondeur et met les connaissances à jour quant aux positions des tribunaux sur le sujet. Elle montre qu'il y a d'abord eu une importante valse-hésitation de la part de la Cour suprême du Canada dans son appréciation des faits en droit constitutionnel. Après une approche initiale formaliste, la Cour suprême a montré une ouverture " certaine mais chaotique " à la réception des faits sociaux. Puis on a assisté à un resserrement des règles quant à leur réception, pour maintenant faire face à un nouveau défi, celui de la "remise en question des postulats factuels du droit" (p. 20).

La partie la plus intéressante du texte est la démonstration par l'auteure que certains "faits sociaux " constituent des postulats essentiels du droit, mais se révèlent des fictions nécessaires dont la remise en question pourrait ébranler beaucoup de certitudes en sol canadien. Elle en donne quelques exemples, notamment celui de la question du droit d'une plaignante en droit criminel de témoigner à visage couvert. On peut se demander s'il existe vraiment un lien fort entre le droit à un procès équitable et la possibilité de voir le visage des témoins. On réalise que la Cour suprême est très divisée sur le sujet. Comme cela arrive souvent, on a préféré ménager la chèvre et le chou plutôt que de trancher le débat. L'auteure écrit très justement ceci (p. 23) : "Une certaine représentation du monde est en effet indispensable à la construction d'un système de règles qui soit logique et cohérent. "Toutefois, ces postulats n'ont pas toujours été vérifiés empiriquement ni considérés au moment de l'élaboration de la norme, ou encore ils ne sont en réalité "qu'une justification fictive ex post facto invoquée au moment de sa contestation " (p. 24).

En somme, l'ouvrage de Pinard est une excellente étude que tout juriste devrait lire et méditer. 